\title{
ON CURVATURES OF SECTIONS OF TENSOR BUNDLES.
}

\author{
P. I. KATSYLO
}

Abstract. We consider natural differential operations acting on sections of tensor vector bundles. Arising problems can be reformulated as invariant theoretical problems (the IT-reduction). We give examples of usage of the IT-reduction. In particular, on a manifold with a connection and a Poisson structure we construct the canonical quantization.

\section{INTRODUCTION.}

Let $M$ be a differentiable $m$-dimensional manifold. The objects of our investigation are natural algebraic differential operations in the form

$$
F_{M}: \Gamma\left(\operatorname{Ass}_{M}(V)\right)^{\mathrm{reg}} \rightarrow \Gamma\left(\operatorname{Ass}_{M}(U)\right),
$$

where $\mathbf{A s s}_{M}(V)$ and $\mathbf{A s s}_{M}(U)$ are tensor bundles, $\Gamma\left(\mathbf{A s s}_{M}(U)\right)$ is the space of differentiable sections of the bundle $\mathbf{A s s}_{M}(U)$, and $\Gamma\left(\mathbf{A s s}_{M}(V)\right)^{\text {reg }}$ is the space of differentiable nondegenerated sections of the bundle $\operatorname{Ass}_{M}(V)$.

A tensor bundle $\mathbf{A s s}_{M}(V)$ is a bundle associated to the frame bundle on $M$ [1], [5]. It corresponds to some linear representation $\rho: \mathbf{G L}(m) \rightarrow \mathbf{G L}(V)$. If

$$
\varphi: M \rightarrow N
$$

is a diffeomorphism of $M$ onto an open submanifold in $N$, then

$$
\varphi^{*}\left(\operatorname{Ass}_{N}(V)\right)=\operatorname{Ass}_{M}(V) .
$$

The typical examples of tensor vector bundles are tangent bundle $T(M)$, cotangent bundle $T^{*}(M), k$-th exterior power of cotangent bundle $\wedge^{k}(M)$ and so on. The nondegeneracy condition is defined by some $r(v) \in \mathbb{R}[V]^{\mathbf{G L}(m)}$. A section $s$ of the bundle $\operatorname{Ass}_{M}(V)$ is called nondegenerated iff for any chart $Y$ we have

$$
s_{Y}(y) \in V^{\text {reg }} \stackrel{\text { def }}{=}\{v \in V \mid r(v) \neq 0\} \subset V
$$

for all $y \in Y$, where $s_{Y}: Y \rightarrow V$ is a presentation of $s$ at the chart $Y$. For example, if $r(v) \equiv$ const $\neq 0$, then every section is nondegenerated.

A differential operation $F_{M}$ is called natural iff for any diffeomorphism

$$
\varphi: M \rightarrow N
$$

of $m$-dimensional manifold $M$ onto an open submanifold of $N$ we have

$$
F_{M}\left(\varphi^{*}(\eta)\right)=\varphi^{*}\left(F_{N}(\eta)\right)
$$

for all $\eta \in \Gamma\left(\operatorname{Ass}_{N}(V)\right)^{\text {reg }}$, where

$$
\varphi^{*}: \Gamma\left(\operatorname{Ass}_{N}(\cdot)\right) \rightarrow \Gamma\left(\operatorname{Ass}_{M}(\cdot)\right)
$$


is an induced by $\varphi$ mapping. This condition is equivalent to the following one. The presentation of $F_{M}$ in bundle charts of $\mathbf{A s s}_{M}(V)$ and $\mathbf{A s s}_{M}(U)$ is written by some universal formulas. These formulas depend on $m$ and on representations $\mathbf{G L}(m): V, U$. The condition that $F_{M}$ is algebraic means that these universal formulas are algebraic. So for any chart $Y \subset M$ we have: $F_{M}\left(s_{Y}\right)$ is a polynomial function in $r\left(s_{Y}\right)^{-1}$ and

$$
\left\{\frac{\partial^{|i|} s_{Y}(y)}{\partial y_{1}^{i_{1}} \ldots \partial y_{m}^{i_{m}}}\right\}_{0 \leq|i| \leq k} .
$$

We consider in this article only algebraic differential operations. We omit the adjective "algebraic" in the sequel.

If $F_{M}$ is a universal differential operation and $s$ is a section of the corresponding tensor bundle, then $F_{M}(s)$ is called a curvature of that section.

The classical example of a natural differential operation is the exterior derivative

$$
d: \Gamma\left(\wedge^{n}(M)\right) \rightarrow \Gamma\left(\wedge^{n+1}(M)\right) .
$$

$d$ is a linear differential operator of order 1 . There is no other natural linear differential operations of order 1 (the Schouten theorem). Here are some other classical curvatures and natural differential operations: the commutator of two vector fields, the curvature of a Riemannian metric, the Laplacian on a Riemannian manifold [1].

In this article we propose the IT-reduction method. This method gives a reduction of problems about natural differential operations to the corresponding invariant theoretical problems. We show how to use the IT-reduction by considering several known problems. By the IT-reduction we obtain in $\$ 3$ a description of curvatures of a Riemannian metric. (This description is known in the classical differential geometry [2]). In \$4 we describe differential operations on a Riemannian manifold. In \$5 we describe curvatures of a connection. In fact, a connection is not a section of any tensor bundle. But one can apply to connections the modified IT-reduction method. In 6 we describe differential operations on a manifold with a connection. As a corollary of this description we obtain in \$7 that for any manifold with a connection $\theta$ and a Poisson structure $\omega$ there exists the canonical quantization $\star(\theta, \omega)$. The word "canonical" means that if $N$ is a manifold with a connection $\theta$ and a Poisson structure $\omega$ and

$$
\varphi: M \rightarrow N
$$

is a diffeomorphism of a manifold $M$ onto an open submanifold of $N$, then

$$
\star\left(\varphi^{*}(\theta), \varphi^{*}(\omega)\right)=\varphi^{*}(\star(\theta, \omega)) .
$$

Finally, we propose the following Conjecture.

Conjecture. Let $M$ be a manifold and $\Gamma\left(\mathbf{A s s}_{M}\left(\wedge^{2} V\right)\right)^{P}$ be a set of all Poisson structures on $M$. Then there are no natural differential operations

$$
\Xi_{k}: \Gamma\left(\mathbf{A s s}_{M}\left(\wedge^{2} V\right)\right)^{P} \times C^{\infty}(M) \times C^{\infty}(M) \rightarrow C^{\infty}(M), \quad k=1,2, \ldots
$$

such that the operation

$$
\begin{array}{r}
\star: C^{\infty}(M) \times C^{\infty}(M) \rightarrow C^{\infty}(M)[[\hbar]], \\
(f, g) \mapsto f g+\Xi_{1}(\omega, f, g) \hbar+\Xi_{2}(\omega, f, g) \hbar^{2}+\ldots
\end{array}
$$


is a quantization of the Poisson structure $\omega$.

Roughly speaking, this conjecture claims that for a canonical quantization of a Poisson structure on a manifold one needs some additional structure on that manifold.

\section{INVARIANT THEORETICAL DESCRIPTION OF NATURAL DIFFERENTIAL OPERATIONS.}

Let $x^{1}, \ldots, x^{m}$ and $z^{1}, \ldots, z^{m}$ be two copies of the standard basis of the space $\mathbb{R}^{m *}$. We consider $x^{1}, \ldots, x^{m}$ as coordinate functions in $\mathbb{R}^{m}$. Consider the linear space $\mathcal{E}_{k}$ of $k$-jets of germs of differentiable functions at $0 \in \mathbb{R}^{m}$. We identify the space $\mathcal{E}_{k}$ and the space of polynomials in the variables $z^{1}, \ldots, z^{m}$ of degree $\leq k$. Consider the group $\mathbf{G L}(m)_{k+1}$ of $(k+1)$-jets of germs of diffeomorphisms of a neighborhood of 0 in $\mathbb{R}^{m}$ onto a neighborhood of 0 in $\mathbb{R}^{m}$. By $\mathbf{G L}(m)_{\infty}$ we denote the group of germs of diffeomorphisms of a neighborhood of 0 in $\mathbb{R}^{m}$ onto a neighborhood of 0 in $\mathbb{R}^{m}$.

We have the canonical group homomorphism

$$
\varphi_{k+1}: \mathbf{G L}(m)_{k+1} \rightarrow \mathbf{G L}(m)
$$

where $\varphi_{k+1}(g)$ is the Jacobi matrix of $g$ at 0 . Set

$$
\mathbf{N}(m)_{k+1}=\left\{g \in \mathbf{G L}(m)_{k+1} \mid \varphi_{k+1}(g)=\mathbf{E}_{m}\right\},
$$

where $\mathbf{E}_{m}$ is the identity matrix of size $m \times m$. The group $\mathbf{G L}(m)_{k+1}$ is a real linear algebraic group, $\mathbf{N}(m)_{k+1}$ is its unipotent radical, and $\mathbf{G L}(m)_{k+1} / \mathbf{N}(m)_{k+1} \simeq \mathbf{G L}(m)$.

Let $\rho: \mathbf{G L}(m) \rightarrow \mathbf{G L}(V)$ be a linear representation. Consider the space $\mathcal{E}_{k} \otimes V$ as a linear space of $k$-jets of germs of differentiable mappings of a neighborhood of 0 in $\mathbb{R}^{m}$ to $V$. By $\mathcal{E}_{\infty} \otimes V$ we denote the linear space of germs of differentiable mappings of a neighborhood of 0 in $\mathbb{R}^{m}$ to $V$. The group $\mathbf{G L}(m)_{\infty}$ acts canonically on the space $\mathcal{E}_{\infty} \otimes V:$

$$
\mathbf{G L}(m)_{\infty}: \mathcal{E}_{\infty} \otimes V, \quad(g \cdot v)(x)=\rho\left(J_{g}\left(g^{-1}(x)\right)\left(v\left(g^{-1}(x)\right)\right),\right.
$$

where $J_{g}(x)$ is the Jacobi matrix of $g$ at $x$. This action corresponds to the transition rule for local presentations of a section of the bundle $\operatorname{Ass}_{M}(V)$ in bundle charts. It defines canonically the action

$$
\begin{array}{r}
\mathbf{G L}(m)_{k+1}: \mathcal{E}_{k} \otimes V, \quad(g \cdot v)(z)= \\
\left\{k \text {-jet of the mapping } x \mapsto \rho\left(J_{g}\left(g^{-1}(x)\right)\left(v\left(g^{-1}(x)\right)\right)\right\},\right.
\end{array}
$$

where $g=g(z) \in \mathbf{G L}(m)_{k+1}, \quad v=v(z) \in \mathcal{E}_{k} \otimes V$.

Note that for $a \geq b$ we have the canonical group homomorphism

$$
\mathbf{G L}(m)_{a} \rightarrow \mathbf{G L}(m)_{b} .
$$

The representation (2.1) and this homomorphism define the representation

$$
\mathbf{G L}(m)_{a}: \mathcal{E}_{b} \otimes V
$$

for all $a>b$.

We have the following $\mathbf{G L}(m)_{k+1}$-equivariant linear mapping

$$
\varepsilon_{k}: \mathcal{E}_{k} \otimes V \rightarrow V, \quad v(z) \mapsto v(0)
$$


Fix $r(v) \in \mathbb{R}[V]^{\mathbf{G L}(m)}$ and consider $\mathbf{G L}(m)$-invariant subset

$$
V^{\text {reg }}=\{v \in V \mid r(v) \neq 0\} \subset V .
$$

This subset defines the natural algebraic nondegeneracy condition for sections of the tensor bundle $\operatorname{Ass}_{M}(V)$. Set

$$
\left(\mathcal{E}_{k} \otimes V\right)^{\mathrm{reg}}=\varepsilon_{k}^{-1}\left(V^{\mathrm{reg}}\right)
$$

Theorem 2.1. Let $\sigma: \mathbf{G L}(m) \rightarrow \mathbf{G L}(U)$ be a linear representation. Then there is the canonical 1-1 correspondence between $\mathbf{G L}(m)_{k+1}$-equivariant morphisms in the form

$$
\alpha:\left(\mathcal{E}_{k} \otimes V\right)^{r e g} \rightarrow U=\mathcal{E}_{0} \otimes U
$$

and the set of natural differential operations of $k$-th order in the form

$$
F_{\alpha, M}: \Gamma\left(\mathbf{A s s}_{M}(V)\right)^{r e g} \rightarrow \Gamma\left(\mathbf{A s s}_{M}(U)\right) .
$$

Proof. First, we show that any $\mathbf{G L}(m)_{k+1}$-equivariant morphism defines canonically a natural differential operation.

Let $\alpha: \mathcal{E}_{k} \otimes V \rightarrow U$ be $\mathbf{G L}(m)_{k+1}$-equivariant morphism. Consider local coordinates $x=\left(x^{1}, \ldots, x^{m}\right)$ in some neighborhood of a point $p \in M$. We assume that the local coordinates defines 1-1 mapping of an open subset $p \in X \subset M$ and some neighborhood $\widetilde{X}$ of 0 in $\mathbb{R}^{m}$. Define the natural differential operation $F_{\alpha, M}$ in the following way. Suppose that $s_{X}: \widetilde{X} \rightarrow V$ is a presentation of a section $s$ of the bundle $\operatorname{Ass}_{M}(V)$ in the chart $X$. We define the section $F_{\alpha, M}(s)$ of the bundle $\operatorname{Ass}_{M}(U)$ in the chart $X$ by the following formula:

$$
\begin{gathered}
\left(F_{\alpha, M}(s)\right)_{X}: \tilde{X} \rightarrow U, \\
x \mapsto \alpha\left(\sum_{0 \leq|i| \leq k} \frac{|i| !}{i_{1} ! \ldots i_{m} !} \frac{\partial^{|i|} s_{X}(x)}{\partial\left(x^{1}\right)^{i_{1}} \ldots \partial\left(x^{m}\right)^{i_{m}}}\left(z^{1}\right)^{i_{1}} \ldots\left(z^{m}\right)^{i_{m}}\right) .
\end{gathered}
$$

Let us check that the section $F_{\alpha, M}(s)$ is well-defined. Let $y=\left(y^{1}, \ldots, y^{m}\right)$ be some other local coordinates in some neighborhood $Y$ of the point $p$. The transition formulas from the local coordinates $x$ to the local coordinates $y$ define a diffeomorphism $g$ of a neighborhood of 0 in $\mathbb{R}^{m}$ onto a neighborhood of 0 in $\mathbb{R}^{m}$. Let $g_{k+1}$ be $(k+1)$-jet of the diffeomorphism $g$ at $0 \in \mathbb{R}^{m}$. Suppose that $s_{Y}: \widetilde{Y} \rightarrow V$ is a presentation of the section $s$ in the chart $Y$. By the transition rule we have

$$
s_{Y}(g(x))=\rho(J(x))\left(s_{X}(x)\right)
$$

where $J(x)$ is the Jacobi matrix of the mapping $g$. We have to check that

$$
\left(F_{\alpha, M}(s)\right)_{Y}(0)=\sigma(J(0))\left(\left(F_{\alpha, M}(s)\right)_{X}(0)\right)
$$


Consider $k$-jets of the left and the right sides of the equation (2.2). We obtain

$$
\begin{gathered}
\left.\sum_{0 \leq|i| \leq k} \frac{|i| !}{i_{1} ! \ldots i_{m} !} \frac{\partial^{|i|} s_{Y}(y)}{\partial\left(y^{1}\right)^{i_{1}} \ldots \partial\left(y^{m}\right)^{i_{m}}}\right|_{y=0}\left(z^{1}\right)^{i_{1}} \ldots\left(z^{m}\right)^{i_{m}}= \\
g_{k+1} \cdot\left(\left.\sum_{0 \leq|i| \leq k} \frac{|i| !}{i_{1} ! \ldots i_{m} !} \frac{\partial^{|i|} s_{X}(x)}{\partial\left(x^{1}\right)^{i_{1}} \ldots \partial\left(x^{m}\right)^{i_{m}}}\right|_{x=0}\left(z^{1}\right)^{i_{1}} \ldots\left(z^{m}\right)^{i_{m}}\right) .
\end{gathered}
$$

By the $\mathbf{G L}(m)_{k+1}$-equivalence of the morphism $\alpha$ we obtain

$$
\begin{gathered}
\left(F_{\alpha, M}(s)\right)_{Y}(0)= \\
\alpha\left(g_{k+1} \cdot\left(\left.\sum_{0 \leq|i| \leq k} \frac{|i| !}{i_{1} ! \ldots i_{m} !} \frac{\partial^{|i|} s_{X}(x)}{\partial\left(x^{1}\right)^{i_{1}} \ldots \partial\left(x^{m}\right)^{i_{m}}}\right|_{x=0}\left(z^{1}\right)^{i_{1}} \ldots\left(z^{m}\right)^{i_{m}}\right)\right)= \\
\sigma(J(0))\left(\alpha\left(\left.\sum_{0 \leq|i| \leq k} \frac{|i| !}{i_{1} ! \ldots i_{m} !} \frac{\partial^{|i|} s_{X}(x)}{\partial\left(x^{1}\right)^{i_{1}} \ldots \partial\left(x^{m}\right)^{i_{m}}}\right|_{x=0}\left(z^{1}\right)^{i_{1}} \ldots\left(z^{m}\right)^{i_{m}}\right)\right)= \\
\sigma(J(0))\left(\left(F_{\alpha, M}(s)\right)_{X}(0)\right) .
\end{gathered}
$$

Conversely, by the construction above any natural differential operation defines canonically the $\mathbf{G L}(m)_{k+1}$-morphism.

Theorem 2.1 reduces the problem of description of natural differential operations of order $k$ acting on $\Gamma\left(\mathbf{A s s}_{M}(V)\right)^{\text {reg }}$ to an invariant theoretical problem. Namely, consider the regular action

$$
\mathbf{N}(m)_{k+1}:\left(\mathcal{E}_{k} \otimes V\right)^{\mathrm{reg}}
$$

and the corresponding algebra of invariants

$$
\mathbb{R}\left[\left(\mathcal{E}_{k} \otimes V\right)^{\mathrm{reg}}\right]^{\mathbf{N}(m)_{k+1}}
$$

We have the canonical representation

$$
\mathbf{G L}(m)_{k+1} / \mathbf{N}(m)_{k+1} \simeq \mathbf{G L}(m): \mathbb{R}\left[\left(\mathcal{E}_{k} \otimes V\right)^{\mathrm{reg}}\right]^{\mathbf{N}(m)_{k+1}}
$$

Every $\mathbf{G L}(m)$-embedding

$$
A_{U}: U \hookrightarrow \mathbb{R}\left[\left(\mathcal{E}_{k} \otimes V\right)^{\mathrm{reg}}\right]^{\mathbf{N}(m)_{k+1}}
$$

of finite dimensional $\mathbf{G L}(m)$-module $U$ defines canonically $\mathbf{G L}(m)_{k+1}$-morphism

$$
\alpha_{U}:\left(\mathcal{E}_{k} \otimes V\right)^{\mathrm{reg}} \rightarrow U^{*}=U^{*} \otimes \mathcal{E}_{0}, \quad \alpha_{U}(v(z))(u)=A_{U}(u)(v(z)) .
$$

Conversely, every $\mathbf{G L}(m)_{k+1}$-morphism (2.4) corresponds to some embedding (2.3) . 


\section{Curvatures of the Riemannian metrics.}

Consider $m$-dimensional manifold $M$. Let $e_{1}, \ldots, e_{m}$ be the standard basis of $\mathbb{R}^{m}$ and $z^{1}, \ldots, z^{m}$ and $u^{1}, \ldots, u^{m}$ are two copies of the dual basis of the dual space $\mathbb{R}^{m *}$. We identify the space $\mathcal{E}_{k}$ and the space of polynomials in the variables $z^{1}, \ldots, z^{m}$ of degree $\leq k$. Riemanian metrics are nondegenerated sections of the bundle $\operatorname{Ass}_{M}\left(S^{2} \mathbb{R}^{m *}\right)$. Fix $k$ and consider the action

$$
\mathbf{N}(m)_{k+1}:\left(\mathcal{E}_{k} \otimes S^{2} \mathbb{R}^{m *}\right)^{\mathrm{reg}} .
$$

In this article we need a simplified concept of the Seshadri section (see [6]).

Definition 3.1. Let $G$ be a linear algebraic group, $G: X$ be a regular action on an affine variety. A closed subvariety $Y \subset X$ is called the nice Seshadri section, if

- $G Y=X$,

- every $G$-orbit intersects transversally $Y$ at one point.

Suppose that $Y \subset X$ is a nice Seshadri section and $V$ is a vector space. Consider $V$ as a trivial $G$-module. Then we have the canonical 1-1 correspondence

$$
\begin{gathered}
\text { \{set of regular mappings of } Y \text { to } V\} \rightarrow \\
\{\text { set of regular } G \text { - mappings of } X \text { to } V\}, \\
\{\hat{\xi}: Y \rightarrow V\} \rightarrow\{\xi: X \rightarrow V, \quad \xi(x)=\hat{\xi}((G \cdot x) \cap Y)\} .
\end{gathered}
$$

Our next purpose is to construct the nice Seshadri section for the action (3.1).

Consider the group $\mathbf{N}(m)_{k+1}$ as an affine variety. Then it is isomorphic to a linear space. Namely, we have the following isomorphism

$$
\begin{aligned}
& \eta:\left(S^{2} \mathbb{R}^{m *} \otimes \mathbb{R}^{m}\right) \times\left(S^{3} \mathbb{R}^{m *} \otimes \mathbb{R}^{m}\right) \times \ldots \times\left(S^{k+1} \mathbb{R}^{m *} \otimes \mathbb{R}^{m}\right) \rightarrow \mathbf{N}(m)_{k+1}, \\
& \left(g_{2}, g_{3}, \ldots, g_{k+1}\right) \mapsto \eta\left(g_{2}, g_{3}, \ldots, g_{k+1}\right)=\left(\mathbf{E}+g_{k+1}\right) \cdot \ldots \cdot\left(\mathbf{E}+g_{3}\right) \cdot\left(\mathbf{E}+g_{2}\right),
\end{aligned}
$$

where $g_{n}=g_{n i}(z) \otimes e_{i} \in S^{n} \mathbb{R}^{m *} \otimes \mathbb{R}^{m}, \quad g_{n i}(z) \in S^{n} \mathbb{R}^{m *}, \quad \mathbf{E}+g_{n}$ is $k$-jet at 0 of the mapping

$$
\mathbb{R}^{m} \rightarrow \mathbb{R}^{m}, \quad c_{i} e_{i} \mapsto\left(c_{i}+g_{n i}\left(c_{1}, \ldots, c_{m}\right)\right) e_{i}
$$

We use the following identification

$$
\begin{gathered}
\left(\mathcal{E}_{k} \otimes S^{2} \mathbb{R}^{m *}\right)^{\mathrm{reg}} \simeq\left(S^{2} \mathbb{R}^{m *}\right)^{\mathrm{reg}} \times\left(\mathbb{R}^{m *} \otimes S^{2} \mathbb{R}^{m *}\right) \times \ldots \times\left(S^{k} \mathbb{R}^{m *} \otimes S^{2} \mathbb{R}^{m *}\right), \\
h=h_{0}+h_{1}+\ldots+h_{k} \sim\left(h_{0}, h_{1}, \ldots, h_{k}\right),
\end{gathered}
$$

where $h_{0} \in\left(S^{2} \mathbb{R}^{m *}\right)^{\text {reg }}, \quad h_{n}=h_{n}(z, u)$ is a bihomogeneous polynomial, $\operatorname{deg}_{z}\left(h_{n}\right)=n$, $\operatorname{deg}_{u}\left(h_{n}\right)=2$, i.e., $h_{n} \in S^{n} \mathbb{R}^{m *} \otimes S^{2} \mathbb{R}^{m *}$.

Suppose

$$
h=\left(h_{0}, h_{1}, \ldots, h_{k}\right) \in\left(\mathcal{E}_{k} \otimes S^{2} \mathbb{R}^{m *}\right)^{\mathrm{reg}} .
$$

For $g=\eta\left(g_{2}, g_{3}, \ldots, g_{k+1}\right) \in \mathbf{N}(m)_{k+1}$ we have

$$
g \cdot h=\left((g \cdot h)_{0},(g \cdot h)_{1}, \ldots,(g \cdot h)_{k}\right),
$$

where $(g \cdot h)_{n}=\mu_{n}\left(\left\{g_{i}, h_{j}\right\}\right)$. From (2.1) it is not difficult to obtain that

$$
\mu_{n}\left(\left\{g_{i}, h_{j}\right\}\right)=\mu_{n}^{\prime}\left(g_{n+1}, h_{0}\right)+\mu_{n}^{\prime \prime}\left(g_{2}, \ldots, g_{n}, h\right)
$$


where

$$
\mu_{n}^{\prime}\left(g_{n+1}, h_{0}\right)=-\frac{\partial^{2} g_{n+1}}{\partial e_{i} \partial z_{j}} \otimes \frac{\partial h_{0}}{\partial u^{i}} u^{j} .
$$

For $n \geq 2$ define $\mathbf{G L}(m)$-submodule

$$
L_{n}=\operatorname{Ker}\left(\delta_{n}\right) \subset S^{n} \mathbb{R}^{m *} \otimes S^{2} \mathbb{R}^{m *},
$$

where

$$
\begin{gathered}
\delta_{n}: S^{n} \mathbb{R}^{m *} \otimes S^{2} \mathbb{R}^{m *} \rightarrow S^{n+1} \mathbb{R}^{m *} \otimes \mathbb{R}^{m *}, \\
\delta_{n}(f(z) \otimes q(u))=f(z) z^{i} \otimes \frac{\partial q(u)}{\partial u^{i}} .
\end{gathered}
$$

The representation $\mathbf{G L}(m): L_{n}$ is irreducible. It is isomorphic to the representation $\mathbf{G L}(m): \mathbf{S}_{(n, 2)}\left(\mathbb{R}^{m *}\right)$, where $\mathbf{S}_{\lambda}$ is the Schur functor corresponding to the partition $\lambda$ (see [3]). Consider the subvariety

$$
\mathbb{L}_{k} \stackrel{\text { def }}{=}\left(S^{2} \mathbb{R}^{m *}\right)^{\mathrm{reg}} \times\{0\} \times L_{2} \times L_{3} \times \ldots \times L_{k} \subset\left(\mathcal{E}_{k} \otimes S^{2} \mathbb{R}^{m *}\right)^{\mathrm{reg}} .
$$

Lemma 3.2. Every $\mathbf{N}(m)_{k+1}$-orbit intersects transversally $\mathbb{L}_{k}$ at one point. In other words, $\mathbb{L}_{k}$ is a nice Seshadri section for the action (3.1).

Proof. Suppose $h \in\left(\mathcal{E}_{k} \otimes S^{2} \mathbb{R}^{m *}\right)^{\text {reg }}$. For $g=\eta\left(g_{2}, g_{3}, \ldots, g_{k+1}\right) \in \mathbf{N}(m)_{k+1}$ the condition $g \cdot h \in \mathbb{L}_{k}$ is equivalent to the equations $\left(E_{1}\right)-\left(E_{k}\right)$, where

$\left(E_{1}\right)\left(\left(\mathbf{E}+g_{2}\right) \cdot h\right)_{1}=0$

$\left.\left(E_{n}\right)\left(\left(\mathbf{E}+g_{n+1}\right) \cdot \ldots \cdot\left(\mathbf{E}+g_{3}\right) \cdot\left(\mathbf{E}+g_{2}\right) \cdot h\right)\right)_{n} \in L_{n}$, where $2 \leq n \leq k$.

We claim that the equations $\left(E_{1}\right)-\left(E_{k}\right)$ for $g$ have a unique solution. More precisely:

(*) One can find sequentially the elements $g_{2}, \ldots, g_{k+1}$ in a unique way from the equations $\left(E_{1}\right), \ldots,\left(E_{k}\right)$ accordingly. Moreover, the equation $\left(E_{n}\right)$ for $g_{n+1}$ with fixed (before defined) $g_{2}, \ldots, g_{n}$ is a linear equation that has a unique solution.

Let us prove $(*)$. First, by the nondegeneracy of $h_{0}$ and the $\mathbf{G L}(m)$-equivalence we can assume that $h_{0}=\left(u^{1}\right)^{2}+\ldots+\left(u^{m}\right)^{2}$.

Consider the equation $\left(E_{1}\right)$ for $g_{2}$. By (3.2) we can rewrite it in the following way:

$$
-2 \frac{\partial^{2} g_{2}}{\partial e_{i} \partial z^{j}} \otimes u^{i} u^{j}+h_{1}=0 .
$$

It is easy to see that this equation for $g_{2}$ is a linear equation having a unique solution.

Suppose that we find $g_{2}, \ldots, g_{n}$ from the equations $\left(E_{1}\right), \ldots,\left(E_{n-1}\right)$. Consider the equation $\left(E_{n}\right)$ for $g_{n+1}$. By (3.2) and the definition of $L_{n}$ we can rewrite the equation $\left(E_{n}\right)$ in the following way:

$$
\delta_{n}\left(-2 \frac{\partial^{2} g_{n+1}}{\partial e_{i} \partial z^{j}} \otimes u^{i} u^{j}+h_{n}^{\prime}\right)=0,
$$

where $h_{n}^{\prime}=h_{n}^{\prime}\left(h, g_{2}, \ldots, g_{n}\right) \in S^{n} \mathbb{R}^{m *} \otimes S^{2} \mathbb{R}^{m *}$. Using the definition of $\delta_{n}$ we simplify the equation (3.3) to the following one.

$$
-2\left(\frac{\partial^{2} g_{n+1}}{\partial e_{i} \partial z^{j}}+\frac{\partial^{2} g_{n+1}}{\partial e_{j} \partial z^{i}}\right) z^{i} \otimes u^{j}+\delta_{n}\left(h_{n}^{\prime}\right)=0 .
$$


It is not difficult to check that the equation (3.4) for $g_{n+1}$ is a linear equation having a unique solution.

Consider the mapping

$$
\widetilde{\alpha}_{k}:\left(\mathcal{E}_{k} \otimes S^{2} \mathbb{R}^{m *}\right)^{\mathrm{reg}} \rightarrow \mathbb{L}_{k}, \quad h \mapsto\left(\mathbf{N}(m)_{k+1} \cdot h\right) \cap \mathbb{L}_{k} .
$$

We have the natural action of the group $\mathbf{G L}(m)$ on $\mathbb{L}_{k}$. This action defines canonically the action $\mathbf{G L}(m)_{k+1}: \mathbb{L}_{k}$ such that the subgroup $\mathbf{N}(m)_{k+1}$ acts on $\mathbb{L}_{k}$ trivially.

For $n=2,3, \ldots, k$ let

$$
\operatorname{pr}_{n}: \mathbb{L}_{k} \rightarrow L_{n}
$$

be the canonical projections. Then

$$
\alpha_{n} \stackrel{\text { def }}{=} \mathbf{p r}_{n} \circ \widetilde{\alpha}_{k}:\left(\mathcal{E}_{k} \otimes S^{2} \mathbb{R}^{m *}\right)^{\text {reg }} \rightarrow L_{n}
$$

is $\mathbf{G L}(m)_{k+1}$-equivariant morphism. By Theorem 2.1 $\alpha_{n}$ corresponds to a natural differential operation

$$
A_{n}: \Gamma\left(\mathbf{A s s}_{M}\left(S^{2} \mathbb{R}^{m *}\right)\right)^{\mathrm{reg}} \rightarrow \Gamma\left(\mathbf{A s s}_{M}\left(L_{n}\right)\right) .
$$

Lemma 3.2 implies the following statement.

Theorem 3.3. Let $U$ be $\mathbf{G L}(m)$-module and

$$
F: \Gamma\left(\operatorname{Ass}_{M}\left(S^{2} \mathbb{R}^{m *}\right)\right)^{r e g} \rightarrow \Gamma\left(\operatorname{Ass}_{M}(U)\right)
$$

be a natural differential operation of order $k$. Then

$$
F(h)=\widetilde{F}\left(\operatorname{det}(h)^{-1}, h, A_{2}(h), \ldots, A_{k}(h)\right),
$$

where $\widetilde{F}$ corresponds to some polynomial $\mathbf{G L}(m)$-mapping

$$
\tilde{f}: \mathbb{R} \times S^{2} \mathbb{R}^{m *} \times L_{2} \times \ldots \times L_{k} \rightarrow U .
$$

Remark 3.4. The classical description of the operation $A_{n}$ is the following: $\left.A_{n}(h)\right|_{p}$ is the homogeneous of degree $n$ summand of the Taylor series of the metric $h$ at the normal local coordinates with center $p$ (see [2]).

\section{NAtUral Differential operations on the RiEmannian MANifolds.}

Theorem 4.1. Let $\rho: \mathbf{G L}(m) \rightarrow \mathbf{G L}(V)$ and $\sigma: \mathbf{G L}(m) \rightarrow \mathbf{G L}(U)$ be linear representations. Then there is the canonical 1-1 correspondence between $\mathbf{G L}(m)$-equivariant differential operations of bounded order in the form

$$
\xi: \mathbb{L}_{k} \times C^{\infty}\left(\mathbb{R}^{m}, V\right) \rightarrow C^{\infty}\left(\mathbb{R}^{m}, U\right),
$$

where $k \in \mathbf{N}$ and the set of natural differential operations of bounded order in the form

$$
\Xi: \Gamma\left(\mathbf{A s s}_{M}(V)\right) \rightarrow \Gamma\left(\mathbf{A s s}_{M}(U)\right)
$$

on a Riemannian manifold. 
Proof. Let $z^{1}, \ldots, z^{m}$ be the standard basis of the space $\mathbb{R}^{m *}$. We identify $\mathcal{E}_{k}$ and the space of polynomials in the variables $z^{1}, \ldots, z^{m}$ of degree $\leq k$.

Suppose we have GL(m)-equivariant differential operation (4.1) of an order $\leq k$. Consider the regular action

$$
\mathbf{N}(m)_{k+1}:\left(\mathcal{E}_{k} \otimes S^{2}\left(\mathbb{R}^{m *}\right)\right)^{\mathrm{reg}} \times\left(\mathcal{E}_{k} \otimes V\right) .
$$

Note that the subvariety $\mathbb{L}_{k} \times\left(\mathcal{E}_{k} \otimes V\right)$ is the nice Seshadri section for this action. Define $\mathbf{G L}(m)$-mapping

$$
\hat{\xi}: \mathbb{L}_{k} \times\left(\mathcal{E}_{k} \otimes V\right) \rightarrow U, \quad(h, v(z)) \mapsto \xi(h, v(z))(0) .
$$

Extend the mapping $\hat{\xi}$ to the $\mathbf{G L}(m)_{k+1}$-mapping

$$
\xi:\left(\mathcal{E}_{k} \otimes S^{2}\left(\mathbb{R}^{m *}\right)\right)^{\mathrm{reg}} \times\left(\mathcal{E}_{k} \otimes V\right) \rightarrow U .
$$

From Theorem 2.1] it follows that $\xi$ defines the natural differential operation

$$
\tilde{\Xi}: \Gamma\left(\mathbf{A s s}_{M}\left(S^{2} \mathbb{R}^{m *}\right)\right)^{\mathrm{reg}} \times \Gamma\left(\mathbf{A s s}_{M}(V)\right) \rightarrow \Gamma\left(\mathbf{A s s}_{M}(U)\right) .
$$

Now suppose that $(M, h)$ is a Riemannian manifold. Define the operation

$$
\Xi: \Gamma\left(\operatorname{Ass}_{M}(V)\right) \rightarrow \Gamma\left(\mathbf{A s s}_{M}(U)\right), \quad s \mapsto \tilde{\Xi}(h, s) .
$$

Conversely, by the construction above any natural differential operation on a Riemannian manifold defines canonically the $\mathbf{G L}(m)$-equivariant differential operation (4.1) of bounded order.

For example, the Laplacian

$$
\Delta: \Gamma\left(\operatorname{Ass}_{M}(V)\right) \rightarrow \Gamma\left(\operatorname{Ass}_{M}(V)\right)
$$

corresponds to the following differential operation

$$
\begin{gathered}
\delta: S^{2}\left(\mathbb{R}^{m *}\right)^{\mathrm{reg}} \times C^{\infty}\left(\mathbb{R}^{m}, V\right) \rightarrow C^{\infty}\left(\mathbb{R}^{m}, V\right), \\
(h, v(x)) \mapsto\left(h^{-1}\right)^{i j} \frac{\partial^{2} v(x)}{\partial x^{i} \partial x^{j}},
\end{gathered}
$$

where $h^{-1} \in S^{2}\left(\mathbb{R}^{m}\right)^{\text {reg }}$ is the dual to $h$ quadratic form.

Remark 4.2. From the proof of Theorem 4.1 we obtain the following description of the corresponding to $\xi$ differential operation $\Xi$. Let $M$ be a Riemannian manifold, $s$ be a section of $\operatorname{Ass}_{M}(V)$, and $p \in M$. Take normal local coordinates with center $p$ and calculate $\Xi(s)(p)$ in that local coordinates by the formula (4.1).

\section{Curvatures of Connections.}

Consider $m$-dimensional manifold $M$. Let $\operatorname{Con}(M)$ be the set of all connections on $M$. In \$1 we define natural differential operations acting on sections of tensor bundles. Analogously one can define natural differential operations acting on connections, pairs $(\theta, \eta)$, where $\theta$ is a connection and $\eta$ is a section of a tensor bundle.

By definition, a curvature of a connection $\theta \in \operatorname{Con}(M)$ is $R(\theta)$, where

$$
R: \operatorname{Con}(M) \rightarrow \Gamma\left(\operatorname{Ass}_{M}(U)\right)
$$

is some natural differential operation. 
In this section we describe curvatures of a connection on a manifold.

Let $e_{1}, \ldots, e_{m}$ be the standard basis of $\mathbb{R}^{m}, x^{1}, \ldots, x^{m}, z^{1}, \ldots, z^{m}, u^{1}, \ldots, u^{m}$, and $v^{1}, \ldots, v^{m}$ be copies of the dual basis of the dual space $\mathbb{R}^{m *}$. We consider $x^{1}, \ldots, x^{m}$ as coordinate functions in $\mathbb{R}^{m}$. Define $\mathcal{E}_{n}, \mathbf{G L}(m)_{n}, \mathbf{N}(m)_{n}$ as in 92 . We identify $\mathcal{E}_{k}$ and the space of polynomials in the variables $z^{1}, \ldots, z^{m}$ of degree $\leq k$.

A connection on $0 \in X \subset \mathbb{R}^{m}$ is a mapping

$$
\theta: X \rightarrow \mathbb{R}^{m} \otimes \mathbb{R}^{m *} \otimes \mathbb{R}^{m *}, \quad x \mapsto \theta(x)=\theta_{i j}^{l}(x) e_{l} \otimes u^{i} \otimes v^{j} .
$$

The mapping $\theta$ defines the connection operator $D_{\theta}$ in the following way:

$$
D_{\theta}\left(e_{i}\right)=\theta_{j i}^{l}(x) e_{l} \otimes u^{j}
$$

Let $\operatorname{Con}(m)_{\infty}$ be the set of germs of connections at $0 \in \mathbb{R}^{m}$ and $\operatorname{Con}(m)_{k}$ be the set of $k$-jets of germs of connections at $0 \in \mathbb{R}^{m}$. We use the following identification

$$
\begin{gathered}
\operatorname{Con}(m)_{k} \simeq\left(\mathbb{R}^{m} \otimes \mathbb{R}^{m *} \otimes \mathbb{R}^{m *}\right) \times\left(\mathbb{R}^{m *} \otimes \mathbb{R}^{m} \otimes \mathbb{R}^{m *} \otimes \mathbb{R}^{m *}\right) \times \\
\left(S^{2}\left(\mathbb{R}^{m *}\right) \otimes \mathbb{R}^{m} \otimes \mathbb{R}^{m *} \otimes \mathbb{R}^{m *}\right) \times \ldots \times\left(S^{k}\left(\mathbb{R}^{m *}\right) \otimes \mathbb{R}^{m} \otimes \mathbb{R}^{m *} \otimes \mathbb{R}^{m *}\right), \\
\theta=\theta_{0}+\theta_{1}+\ldots+\theta_{k} \sim\left(\theta_{0}, \theta_{1}, \ldots, \theta_{k}\right),
\end{gathered}
$$

where $\theta_{n}=\theta_{n}(z, e, u, v)$ is a polyhomogeneous polynomial of polydegree $(n, 1,1,1)$, i.e., $\theta_{n} \in S^{n}\left(\mathbb{R}^{m *}\right) \otimes \mathbb{R}^{m} \otimes \mathbb{R}^{m *} \otimes \mathbb{R}^{m *}$.

The group $\mathbf{G L}(m)_{\infty}$ acts canonically on $\operatorname{Con}(m)_{\infty}$ :

$$
\begin{gathered}
\mathbf{G L}(m)_{\infty}: \operatorname{Con}(m)_{\infty} \\
(g * \theta)(x)=\left(J_{g}(x)_{a}^{l} \frac{\partial J_{g}^{-1}(x)_{j}^{a}}{\partial x^{i}}+J_{g}(x)_{a}^{l} \theta_{i b}^{a}(x) J_{g}^{-1}(x)_{j}^{b}\right) e_{l} \otimes u^{i} \otimes v^{j}
\end{gathered}
$$

where $J_{g}(x)$ is the Jacobi matrix of $g$ at $x$. This action corresponds to the transition rule for local presentations of a connection in charts. It defines canonically the action

$$
\begin{gathered}
\mathbf{G L}(m)_{k+2}: \operatorname{Con}(m)_{k}, \quad(g \cdot \theta)(z)=\{k-\text { jet of the mapping } \\
\left.x \mapsto\left(J_{g}(x)_{a}^{l} \frac{\partial J_{g}^{-1}(x)_{j}^{a}}{\partial x^{i}}+J_{g}(x)_{a}^{l} \theta_{i b}^{a}(x) J_{g}^{-1}(x)_{j}^{b}\right) e_{l} \otimes u^{i} \otimes v^{j}\right\},
\end{gathered}
$$

where $g=g(z) \in \mathbf{G L}(m)_{k+2}, \quad \theta=\theta(z) \in \mathbf{C o n}(m)_{k}$. The action $\mathbf{G L}(m)_{k+2}: \operatorname{Con}(m)_{k}$ is an affine action. Consider the restriction of this action to the subgroup $\mathbf{N}(m)_{k+2} \subset$ $\mathbf{G L}(m)_{k+2}$ :

$$
\mathbf{N}(m)_{k+2}: \operatorname{Con}(m)_{k}
$$

Theorem 5.1. Let $\rho: \mathbf{G L}(m) \rightarrow \mathbf{G L}(V)$ and $\sigma: \mathbf{G L}(m) \rightarrow \mathbf{G L}(U)$ be linear representations. Then there is the canonical 1-1 correspondence between $\mathbf{G L}(m)_{k+2}$-morphisms in the form

$$
\alpha: \operatorname{Con}(m) \times\left(\mathcal{E}_{k} \otimes V\right) \rightarrow U=\mathcal{E}_{0} \otimes U
$$

and the set of natural differential operations of order $k$ in the form

$$
F_{\alpha, M}: \operatorname{Con}(M) \times \Gamma\left(\operatorname{Ass}_{M}(V)\right) \rightarrow \Gamma\left(\operatorname{Ass}_{M}(U)\right) .
$$


The proof of this Theorem is the same as of Theorem 2.1.

Our next purpose is to construct the nice Seshadri section for the action (5.2).

We use the isomorphism $\eta$ from the section 3 . Suppose

$$
\theta=\left(\theta_{0}, \theta_{1}, \ldots, \theta_{k}\right) \in \operatorname{Con}(m)_{k},
$$

where $\theta_{n} \in S^{n}\left(\mathbb{R}^{m *}\right) \otimes \mathbb{R}^{m} \otimes \mathbb{R}^{m *} \otimes \mathbb{R}^{m *}$. For $g=\eta\left(g_{2}, g_{3}, \ldots, g_{k+2}\right) \in \mathbf{N}(m)_{k+2}$ we have

$$
g \cdot \theta=\left((g \cdot \theta)_{0},(g \cdot \theta)_{1}, \ldots,(g \cdot \theta)_{k}\right),
$$

where $(g \cdot h)_{n}=\nu_{n}\left(\left\{g_{i}, h_{j}\right\}\right)$. From (5.1) it is easy to obtain that

where

$$
\nu_{n}\left(\left\{g_{i}, \theta_{j}\right\}\right)=\nu_{n}^{\prime}\left(g_{n+2}\right)+\nu_{n}^{\prime \prime}\left(g_{2}, \ldots, g_{n+1}, \theta\right)
$$

Define $\mathbf{G L}(m)$-submodule

$$
\nu_{n}^{\prime}\left(g_{n+2}\right)=-\frac{\partial^{2} g_{n+2}}{\partial z^{i} \partial z^{j}} \otimes u^{i} \otimes v^{j}
$$

$$
C_{n}=\operatorname{Ker}\left(\gamma_{n}\right) \subset S^{n}\left(\mathbb{R}^{m *}\right) \otimes \mathbb{R}^{m} \otimes \mathbb{R}^{m *} \otimes \mathbb{R}^{m *},
$$

where

$$
\begin{gathered}
\gamma_{n}: S^{n}\left(\mathbb{R}^{m *}\right) \otimes \mathbb{R}^{m} \otimes \mathbb{R}^{m *} \otimes \mathbb{R}^{m *} \rightarrow S^{n+2} \mathbb{R}^{m *} \otimes \mathbb{R}^{m}, \\
\gamma_{n}\left(f(z) \otimes e_{l} \otimes u^{i} \otimes v^{j}\right)=f(z) z^{i} z^{j} \otimes e_{l} .
\end{gathered}
$$

Consider the subvariety

$$
\mathbb{C}_{k} \stackrel{\text { def }}{=} C_{0} \times C_{1} \times \ldots \times C_{k} \subset \operatorname{Con}(m)_{k} .
$$

Lemma 5.2. Every $\mathbf{N}(m)_{k+2}$-orbit intersects transversally $\mathbb{C}_{k}$ at one point. In other words, $\mathbb{C}_{k}$ is a nice Seshadri section for the action (5.2).

Proof. Suppose $\theta \in \operatorname{Con}(m)_{k}$. For $g=\eta\left(g_{2}, g_{3}, \ldots, g_{k+2}\right) \in \mathbf{N}(m)_{k+2}$ the condition $g \cdot \theta \in \mathbb{C}_{k}$ is equivalent to equations $\left(D_{0}\right)-\left(D_{k}\right)$, where

$$
\left.\left(D_{n}\right)\left(\left(\mathbf{E}+g_{n+2}\right) \cdot \ldots \cdot\left(\mathbf{E}+g_{3}\right) \cdot\left(\mathbf{E}+g_{2}\right) \cdot \theta\right)\right)_{n} \in C_{n} .
$$

It is easy to see that the Lemma is a corollary of the following claim.

(*) One can find sequentially the elements $g_{2}, \ldots, g_{k+2}$ in a unique way from the equations $\left(D_{0}\right), \ldots,\left(D_{k}\right)$ accordingly. Moreover, the equation $\left(D_{n}\right)$ for $g_{n+2}$ with fixed (before defined) $g_{2}, \ldots, g_{n+1}$ is a linear equation that has a unique solution.

Let us prove claim (*). By (5.3) and the definition of $C_{n}$ we can rewrite the equation $\left(D_{n}\right)$ in the following way:

$$
\gamma_{n}\left(-\frac{\partial^{2} g_{n+2}}{\partial z^{i} \partial z^{j}} \otimes u^{i} \otimes v^{j}+\theta_{n}^{\prime}\right)=0,
$$

where $\theta_{n}^{\prime}=\theta_{n}^{\prime}\left(\theta, g_{2}, \ldots, g_{n+1}\right) \in S^{n}\left(\mathbb{R}^{m *}\right) \otimes \mathbb{R}^{m} \otimes \mathbb{R}^{m *} \otimes \mathbb{R}^{m *}$. Using the definition of $\gamma_{n}$ and the Euler theorem about homogeneous functions we get

$$
-(n+2)(n+1) g_{n+2}+\gamma_{n}\left(\theta_{n}^{\prime}\right)=0 .
$$

It is clear that this equation for $g_{n+2}$ with a fixed (before defined) $g_{2}, \ldots, g_{n+1}$ is a linear equation having a unique solution. 
Consider the mapping

$$
\widetilde{\psi}_{k}: \operatorname{Con}(m)_{k} \rightarrow \mathbb{C}_{k}, \quad \theta \mapsto\left(\mathbf{N}(m)_{k+2} \cdot \theta\right) \cap \mathbb{C}_{k}
$$

The natural action of the group $\mathbf{G L}(m)$ on $\mathbb{C}_{k}$ defines canonically the action $\mathbf{G L}(m)_{k+2}$ : $\mathbb{C}_{k}$ such that the subgroup $\mathbf{N}(m)_{k+2}$ acts trivially. For $n=0,1, \ldots, k$ let

$$
\operatorname{pr}_{n}: \mathbb{C}_{k} \rightarrow C_{n}
$$

be canonical projections. Then

$$
\psi_{n} \stackrel{\text { def }}{=} \mathbf{p r}_{n} \circ \widetilde{\psi}_{k}: \operatorname{Con}(m)_{k} \rightarrow C_{n}
$$

is $\mathbf{G L}(m)_{k+2}$-morphism. From Theorem 5.1 it follows that $\psi_{n}$ defines canonically a natural differential operation

$$
\Psi_{n}: \operatorname{Con}(M) \rightarrow \Gamma\left(\operatorname{Ass}_{M}\left(C_{k}\right)\right) .
$$

From Lemma 5.2 we obtain the following statement.

Theorem 5.3. Let $U$ be a $\mathbf{G L}(m)$-module and

$$
F: \operatorname{Con}(M) \rightarrow \Gamma\left(\mathbf{A s s}_{M}(U)\right)
$$

be a natural differential operation of order $k$. Then

$$
F(\theta)=\widetilde{F}\left(\Psi_{0}(\theta), \ldots, \Psi_{k}(\theta)\right),
$$

where $\widetilde{F}$ corresponds to some polynomial $\mathbf{G L}(m)$-mapping

$$
\tilde{f}: C_{0} \times C_{1} \times \ldots \times C_{k} \rightarrow U \text {. }
$$

Remark 5.4. From the construction of $\Psi_{n}$ we obtain the following geometrical description of the curvature $\left.\Psi_{n}(\theta)\right|_{p}$ of the connection $\theta$ on $M$ at $p \in M$. Take local coordinates $\left(x^{1}, \ldots, x^{m}\right)$ with center $p$ such that for the Taylor series

$$
\theta=\theta_{0}+\theta_{1}+\theta_{2}+\ldots
$$

of the connection $\theta$ in the local coordinates $\left(x^{1}, \ldots, x^{m}\right)$ we have: $\theta_{l} \in C_{l}$ for all $0 \leq l \leq n$. Then the local presentation at $p$ in the local coordinates $\left(x^{1}, \ldots, x^{m}\right)$ of the curvature $\Psi_{n}(\theta)$ is $\theta_{n}$.

Remark 5.5. It is easy to see that $\Psi_{0}(\theta)$ is the torsion of the connection $\theta$.

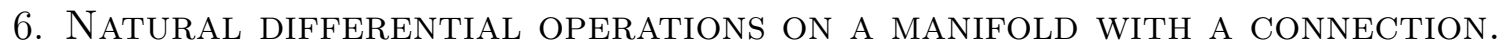

Theorem 6.1. Let $\rho: \mathbf{G L}(m) \rightarrow \mathbf{G L}(V)$ and $\sigma: \mathbf{G L}(m) \rightarrow \mathbf{G L}(U)$ be linear representations. Then there is the canonical 1-1 correspondence between $\mathbf{G L}(m)$-equivariant differential operations of bounded order in the form

$$
\xi: \mathbb{C}_{k} \times C^{\infty}\left(\mathbb{R}^{m}, V\right) \rightarrow C^{\infty}\left(\mathbb{R}^{m}, U\right),
$$

where $k \in \mathbf{N}$ and the set of natural differential operations of bounded order in the form

$$
\Xi: \Gamma\left(\mathbf{A s s}_{M}(V)\right) \rightarrow \Gamma\left(\mathbf{A s s}_{M}(U)\right)
$$

on a manifold with a connection. 
Proof. Let $z^{1}, \ldots, z^{m}$ be the standard basis of the space $\mathbb{R}^{m *}$. We identify $\mathcal{E}_{k}$ and the space of polynomials in the variables $z^{1}, \ldots, z^{m}$ of degree $\leq k$.

Suppose we have $\mathbf{G L}(m)$-equivariant differential operations (6.1) of an order $\leq k$. Consider the regular action

$$
\mathbf{N}(m)_{k+2}: \operatorname{Con}(m)_{k} \times\left(\mathcal{E}_{k} \otimes V\right) .
$$

Note that the subvariety $\mathbb{C}_{k} \times\left(\mathcal{E}_{k} \otimes V\right)$ is a nice Seshadri section for this action. Define $\mathbf{G L}(m)$-mapping

$$
\hat{\xi}: \mathbb{C}_{k} \times\left(\mathcal{E}_{k} \otimes V\right) \rightarrow U, \quad(\theta, v(z)) \mapsto \xi(\theta, v(z))(0) .
$$

Extend the mapping $\hat{\xi}$ to the $\mathbf{G L}(m)_{k+2}$-mapping

$$
\xi: \operatorname{Con}(m)_{k} \times\left(\mathcal{E}_{k} \otimes V\right) \rightarrow U,
$$

From Theorem 5.1 it follows that $\xi$ defines a natural differential operation

$$
\tilde{\Xi}: \operatorname{Con}(M) \times \Gamma\left(\operatorname{Ass}_{M}(V)\right) \rightarrow \Gamma\left(\operatorname{Ass}_{M}(U)\right) .
$$

Now suppose that $(M, \theta)$ is a manifold with a connection. Define the operation

$$
\Xi: \Gamma\left(\mathbf{A s s}_{M}(V)\right) \rightarrow \Gamma\left(\mathbf{A s s}_{M}(U)\right), \quad s \mapsto \tilde{\Xi}(\theta, s) .
$$

Conversely, by the construction above any natural differential operation of bounded order on a manifold with a connection defines canonically $\mathbf{G L}(m)$-equivariant differential operation (6.1) of bounded order.

Remark 6.2. From the proof of Theorem 4.1 we obtain the following description of the corresponding to $\xi$ differential operation $\Xi$. Let $M$ be a manifold with a connection $\theta, s$ be a section of $\operatorname{Ass}_{M}(V)$, and $p \in M$. Take local coordinates $\left(x^{1}, \ldots, x^{m}\right)$ with center $p$ such that for the Taylor series $\theta=\theta_{0}+\theta_{1}+\ldots$ of the connection $\theta$ in the local coordinates $\left(x^{1}, \ldots, x^{m}\right)$ we have: $\theta_{l} \in C_{l}$ for all $0 \leq l \leq n$. Now calculate $\Xi(s)(p)$ in that local coordinates by the formula (6.1).

\section{Canonical quantization of the Poisson structures on a manifold WITH A CONNECTION.}

We need $\star(\omega)$-product in $\mathbb{R}^{m}$.

Definition 7.1. Let $x=\left(x^{1}, \ldots, x^{m}\right)$ be coordinate functions in $\mathbb{R}^{m}$ and

$$
\omega=\omega(x)=\omega^{i j}(x) \frac{\partial}{\partial x^{i}} \wedge \frac{\partial}{\partial x^{j}}
$$

be a Poisson structure. $\star(\omega)$-product in $\mathbb{R}^{m}$ is a mapping

$$
\begin{gathered}
\star(\omega): C^{\infty}\left(\mathbb{R}^{m}\right) \times C^{\infty}\left(\mathbb{R}^{m}\right) \rightarrow C^{\infty}\left(\mathbb{R}^{m}\right)[[\hbar]], \\
\star(\omega)(f, g)=f g+\beta_{1}(\omega, f, g) \hbar+\beta_{2}(\omega, f, g) \hbar^{2}+\ldots
\end{gathered}
$$

such that

- $\star(\omega)$ is a quantization of the Poisson structure $\omega$, 


$$
\beta_{k}: C^{\infty}\left(\mathbb{R}^{m}, \wedge^{2}\left(\mathbb{R}^{m}\right)\right) \times C^{\infty}\left(\mathbb{R}^{m}\right) \times C^{\infty}\left(\mathbb{R}^{m}\right) \rightarrow C^{\infty}\left(\mathbb{R}^{m}\right)
$$

is $\mathbf{G L}(m)$-equivariant differential operation of bounded order, where $k=1,2, \ldots$.

$\star(\omega)$-product in $\mathbb{R}^{m}$ is a natural generalization of the Moyal $\star$-product. The first $\star(\omega)$-product in $\mathbb{R}^{m}$ was constructed by Kontsevich in [4].

Remark 7.2. By using the IT-reduction it is not difficult to prove that there exists $\star(\omega)$ product in $\mathbb{R}^{m}$. This proof is constructive: it gives an algorithm for calculation the operations $\beta_{k}$.

Theorem 7.3. For a manifold with a connection and a Poisson structure there exists the canonical quantization.

Proof. Let $M$ be a manifold with a connection $\theta$ and a Poisson structure $\omega$. Let the natural differential operation

$$
B_{k}: \Gamma\left(\operatorname{Ass}_{M}\left(\wedge^{2} \mathbb{R}^{m}\right)\right) \times C^{\infty}(M) \times C^{\infty}(M) \rightarrow C^{\infty}(M)
$$

corresponds to $\beta_{k}$, where $k=1,2, \ldots$ (see Theorem 6.1). Set

$$
\begin{array}{r}
\star: C^{\infty}(M) \times C^{\infty}(M) \rightarrow C^{\infty}(M)[[\hbar]], \\
(f, g) \mapsto f g+B_{1}(\omega, f, g) \hbar+B_{2}(\omega, f, g) \hbar^{2}+\ldots
\end{array}
$$

We claim that the operation (17.1) is a quantization of the Poisson structure $\omega$. To prove it we take a point $p \in M$ and $n \in \mathbf{N}$ and prove that the defined above operation $\star$ gives a quantization of the Poisson structure $\omega$ modulo $O\left(\hbar^{n+1}\right)$. Suppose that $\left(x^{1}, \ldots, x^{m}\right)$ are local coordinates with center $p$ such that for the Taylor series $\theta=\theta_{0}+\theta_{1}+\ldots$ of the connection $\theta$ in the local coordinates $\left(x^{1}, \ldots, x^{m}\right)$ we have: $\theta_{l} \in C_{l}$ for all $0 \leq l \leq n$. By Remark 6.2 the operation $\star$ at the point $p$ in the local coordinates $\left(x^{1}, \ldots, x^{m}\right)$ coincides with the Moyal-Kontsevich $\star(\omega)$-product modulo $O\left(\hbar^{n+1}\right)$. This concludes the proof.

Remark 7.4. From section 4 one can easily obtain the following quantization rule for a Riemannian manifold.

Let $M$ be a Riemannian manifold with a Poisson structure $\omega, p \in M$. Take normal local coordinates $\left(x^{1}, \ldots, x^{m}\right)$ with center $p$. Then define $\star$-product at $p$ in coordinates $\left(x^{1}, \ldots, x^{m}\right)$ by the Moyal-Kontsevich formula for $\star(\omega)$-product.

\section{REFERENCES}

[1] Besse A., Einstein manifolds. Springer-Verlag, 1987.

[2] Epstein D.B.A., Natural tensors on Riemannian manifolds J. Diff. Geom., 1975, 10, p. $631-645$.

[3] Fulton W., Harris J., Representation Theory. Springer-Verlag, 1991.

[4] Kontsevich M., Deformation quantization of Poisson manifolds I. QA/9709040.

[5] Nomizu K., Lie Groups and Differential Geometry. Mathematical Society of Japan, Tokyo, 1956.

[6] Seshadri C.S., On a theorem of Weitzenbök in invariant theory J. Math. Kyoto Univ., 1962, 1, p. 403-409.

Independent University of Moscow, Bolshoi Vlasievskit, 11, 117463, Moscow E-mail address: katsylo@katsylo.mccme.ru 